\title{
SYNTHESIS OF MICROSTRUCTURE-INFORMED ENRICHMENT FUNCTIONS BY MEANS OF WANG TILES
}

\begin{abstract}
M. Doškáŕr ${ }^{*}$, J. Novák $^{* *}$, J. Zeman ${ }^{* * *}$
Abstract: Wang tiles-based description of a microstructural geometry of random heterogeneous materials facilitates fast reconstruction of material samples. As a step towards a statistical quantification of the microstructure influence on the response of macro-scale models with fully resolved microstructural geometry, we present a framework that accelerates the numerical analyses by (i) pre-computing characteristic responses of the compressed microstructural representation and (ii) plugging these responses as microstructure-informed enrichments into Extended Finite Element Method. The methodology is illustrated with a scalar steady-state problem.
\end{abstract}

\section{Keywords: Wang tiling, microstructure synthesis, microstructure-informed enrichment functions}

\section{Introduction}

Composition of a material microstructure, along with properties of its constituents, determines the material's macroscopic behaviour. Current trends in numerical modelling thus focus on efficient methods that integrate knowledge of material microstructure in order to increase their predictive ability. For instance, computational homogenization replaces elaborate macroscopic constitutive laws with a boundary value problem solved for a representative sample (often called PUC) of the material microstructure.

In our previous works (Novák et al., 2012; Doškář et al., 2014), we have presented an extension of PUC based on the abstract concept of Wang tiles for modelling random heterogeneous materials. Our extension enables efficient synthesis of stochastic microstructural realizations, allowing us to generate macroscopic models with fully resolved microstructural geometry. In order to achieve our ultimate goal of statistical quantification of a macroscopic response under microstructural randomness, the fast synthesis must be complemented with equally efficient numerical schemes that exploit the compressed nature of the microstructural geometry. Here, we present a framework that (i) extracts characteristic responses of the compressed microstructure, see Section 2, and (ii) re-use them as microstructure-informed enrichments in Extended Finite Element Method, Section 3.

In the sequel, we illustrate the framework with a scalar elliptic problem (represented here with a linear, steady-state heat conduction) governed by

$$
\nabla \cdot(-\boldsymbol{K}(\boldsymbol{x}) \cdot \nabla \theta(\boldsymbol{x}))=0, \quad \forall \boldsymbol{x} \in \Omega,
$$

where $\theta$ denotes the temperature field and $\boldsymbol{K}$ is a second-order conductivity tensor mediating a linear constitutive relation $\boldsymbol{q}=-\boldsymbol{K} \cdot \nabla \theta$ between the heat flux $\boldsymbol{q}$ and the temperature gradient $\nabla \theta$. Governing equation (1) is further supplemented with boundary conditions:

$$
\theta(\boldsymbol{x})=\hat{\theta}(\boldsymbol{x}), \boldsymbol{x} \in \Gamma_{\theta} \neq \emptyset, \quad \text { and } \quad \boldsymbol{n}(\boldsymbol{x}) \cdot \boldsymbol{q}(\boldsymbol{x})=\hat{q}(\boldsymbol{x}), \boldsymbol{x} \in \Gamma_{\boldsymbol{q}},
$$

where $\hat{\theta}$ and $\hat{q}$ are a given temperature and normal heat flux profiles prescribed at boundary parts $\Gamma_{\theta}$ and $\Gamma_{\boldsymbol{q}}$ of the domain $\Omega$.

Ing. Martin Doškář: Faculty of Civil Engineering, Czech Technical University in Prague, Thákurova 2077/7, Prague; CZ, martin.doskar@fsv.cvut.cz

** Ing. Jan Novák, Ph.D.: Faculty of Civil Engineering, Czech Technical University in Prague, Thákurova 2077/7, Prague; CZ, novakja@fsv.cvut.cz

**** Assoc. Prof. Jan Zeman, Ph.D.: Faculty of Civil Engineering, Czech Technical University in Prague, Thákurova 2077/7, Prague; CZ, Jan.Zeman@cvut.cz 


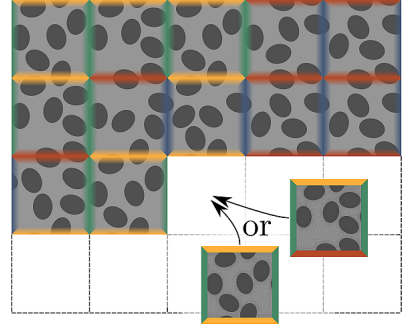

(a)
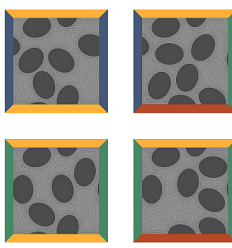

(b)
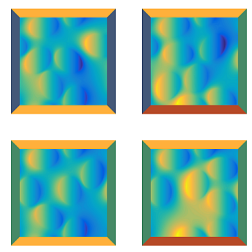

(c)

Fig. 1: Illustration of: (a) a tiling and a step in the assembly algorithm, (b) a tile set, and (c) a pre-computed enrichment field.

\section{Microstructure-informed enrichment functions}

Assume that a microstructural geometry under investigation is compressed within a set $\mathcal{S}$ of $n_{\mathcal{T}}$ Wang tiles-square domains $\mathcal{T}_{(i)}$ with pre-defined mutual compatibility illustrated with colour codes in Fig. 1b. The tile codes are designed such that a random realization of the microstructure can be assembled with a simple stochastic algorithm: an initially empty grid is sequentially traversed, a subset of tiles compliant with compatibility constraints posed by previously placed tiles is identified at each grid node, and one tile is randomly chosen from the subset and placed. Albeit stochastic the resulting microstructural sample consists of a limited number of repeating subdomains.

Pre-computing characteristic responses of the compressed system was first proposed by Novák et al. (2013), who incorporated traction jumps across corresponding tile edges into the objective function used for designing the compressed set. Depending on the weight attributed to the traction jumps, the resulting tile morphology either exhibited periodic arrangement (for a large weight) or featured lingering traction jumps. Here, we pre-compute tile responses that are continuous for already designed tile representations.

Similarly to numerical homogenization, we split the primary unknown $\theta(\boldsymbol{x})=\theta^{*}(\boldsymbol{x})+\boldsymbol{G} \cdot \boldsymbol{x}$ into a fluctuation part $\theta^{*}$, reflecting the microstructural heterogeneity, and a macroscopic part governed by a prescribed gradient $G$, which plays the role of loading. Without loss of generality, we assume that the tile domains are centred, $\mathcal{T}_{(i)}=[-1,1]^{2}, \forall i=1 \ldots n_{\mathcal{T}}$. Continuity of the fluctuation field $\theta^{*}$ is trivially ensured by assigning the same unknowns to corresponding tile edges. Assembling the stiffness matrix of the whole set, we arrive at

$$
\left[\begin{array}{ccc}
\mathrm{K}^{\mathcal{S}} & \mathrm{C}_{\mathrm{I}}^{\top} & \mathrm{C}_{\mathrm{II}}^{\mathrm{T}} \\
\mathrm{C}_{\mathrm{I}} & 0 & 0 \\
\mathrm{C}_{\mathrm{II}} & 0 & 0
\end{array}\right]\left\{\begin{array}{c}
\theta^{*} \\
\lambda_{\mathrm{I}} \\
\lambda_{\mathrm{II}}
\end{array}\right\}=\left\{\begin{array}{c}
\mathrm{f}(\boldsymbol{G}) \\
0 \\
0
\end{array}\right\},
$$

where the additional constraint $\mathrm{C}_{\mathrm{I}} \theta^{*}=0 \Leftrightarrow \int_{\mathcal{S}} \theta^{*}(\boldsymbol{x}) \mathrm{d} \boldsymbol{x}=0$ handles the zero energy modes of the problem and $\mathrm{C}_{\mathrm{II}} \theta^{*}=0$ corresponds to one of the following conditions that prevent $\theta^{*}$ from compensating for the prescribed loading:

$$
\begin{aligned}
\left.\theta^{*}\right|_{\partial \mathcal{T}_{(i)}} & =0, \quad \forall i=1 \ldots n_{\mathcal{T}}, \\
\int_{\partial \mathcal{T}_{(i)}} \theta^{*}(\boldsymbol{x}) \boldsymbol{n}(\boldsymbol{x}) \mathrm{d} S & =\mathbf{0}, \quad \forall i=1 \ldots n_{\mathcal{T}}, \\
\int_{\partial \mathcal{S}} \theta^{*}(\boldsymbol{x}) \boldsymbol{n}(\boldsymbol{x}) \mathrm{d} S & =\mathbf{0} .
\end{aligned}
$$

Solving Eq. (3) yields a fluctuation field $\theta^{*}$ for a particular choice of the second constraint and prescribed macroscopic gradient $\boldsymbol{G}$. Due to linearity of the illustrative problem, only two cases $\boldsymbol{G}=\left[\begin{array}{ll}1 & 0\end{array}\right]^{\top}$ and $\boldsymbol{G}=\left[\begin{array}{ll}0 & 1\end{array}\right]^{\top}$ suffice. Combined with three types of $\mathrm{C}_{\mathrm{II}}$, six enrichment fields are pre-computed. Constraints $(\mathrm{K}),(\mathrm{P})$, and $(\mathrm{S})$ loosely correspond to the boundary conditions traditionally employed in numerical homogenization; however, unlike in numerical homogenization, we combine them to enrich the approximation space. 


\section{Reduced model}

Assume a macroscopic model whose microstructural geometry is provided by means of Wang tiles and consider the elliptic problem governed by Eq. (1). A numerical solution to the problem follows from the standard weak form:

$$
\text { Find } \theta \in \mathcal{U} \text { such that } a(\theta, \vartheta)=b(\vartheta), \forall \vartheta \in \mathcal{U}_{0} \text {, }
$$

where $\mathcal{U}$ denotes the space of admissible fields $\theta(\boldsymbol{x})$ and $\mathcal{U}_{0}$ is the counterpart that vanishes at $\Gamma_{\theta} ; a(\bullet, \bullet)$ and $b(\bullet)$ stand for the bilinear and linear form, respectively, arising from Eq. (1).

Quality of a numerical approximation is primarily determined by the fitness of a finite-dimensional subspace $\mathcal{U}^{h} \in \mathcal{U}$. In the Finite Element (FE) formulation, the approximation space consists of polynomial functions defined element-wise; its quality can be thus controlled with the characteristic element size $h$ and/or the polynomial order. For the elliptic problem considered in our work, the presence of microstructural geometry in the macroscopic model requires the finite-element discretization to adequately resolve microstructural traits, resulting in a high-dimensional approximation space.

A remedy for high-dimensionality rests on enriching the approximation space with a priori knowledge of local character of the solution. In Extended Finite Element Method (XFEM), these enrichments are plugged into the model following the partition-of-unity idea.The numerical solution $\theta^{r}(\boldsymbol{x})$ then takes the form:

$$
\theta^{r}(\boldsymbol{x})=\sum_{i} N_{i}(\boldsymbol{x}) u_{i}+\sum_{j} \sum_{k} N_{j}(\boldsymbol{x}) \psi_{k}(\boldsymbol{x}) v_{k}^{j},
$$

where $N_{i}(\boldsymbol{x})$ are the usual FE shape functions, $\psi_{j}(\boldsymbol{x})$ denotes an enrichment, and $u_{i}$ and $v_{k}^{j}$ are the standard and enriched Degrees of Freedom (DOFs), respectively. XFEM allows for successful modelling of crack propagation without remeshing.

In modelling heterogeneous materials, similar ideas were adopted by Strouboulis et al. (2003), who precomputed numerical "handbook" functions for closely packed inclusions. Recently, Plews and Duarte (2015) introduced a mixed approach combining XFEM with results computed for a patches of fully-resolved microstructural geometry. While we advance on the previous two approaches, we differ in an important aspect of re-usability of the enrichments. Unlike (Strouboulis et al., 2003; Plews and Duarte, 2015), who rely on solving local boundary values problem just prior or during the analysis and handle only the specific macroscopic geometry and loading, we pre-calculate responses of the compressed microstructure for any geometry and loading.

Our approach proceed in the spirit of XFEM: we construct a coarse FE mesh over the model domain, irrespectively of the microstructural phase boundaries, and supplement the approximation space with microstructure-informed enrichment functions assembled from the pre-computed tile responses. The standard DOFs (the first sum in the right-hand side of Eq. (5)) thus cover the global character of the solution while the enriched DOFs (the second sum in Eq. (5)) capture fluctuations in the solution caused by the presence of heterogeneities. Plugging the XFEM approximation (5) into Eq. (4) yields the standard linear system of equations $\mathrm{K}^{r} \mathrm{u}^{r}=\mathrm{f}^{r}$, where $\mathrm{u}^{r}$ collects all DOFs from Eq. (5). A common drawback of XFEM arises from computing entries of $\mathrm{K}^{r}$ and $\mathrm{f}^{r}$. In our particular case, the enrichments are calculated for a fine discretization at the level of individual tiles and oscillate rapidly, which prohibits use of common quadrature formulas.

To simplify the implementation, we adopt the viewpoint of Reduced Order Modelling. We re-use the FE meshes defined for each tile to construct discretization of the whole macroscopic problem, resulting in a high-dimensional approximation space $\mathcal{U}^{f}$ and the system $\mathrm{K}^{f} \mathrm{u}^{f}=\mathrm{f}^{f}$. Next, we take Eq. (5) as a definition of reduced modes $q_{i}(\boldsymbol{x})$, spanning the subspace $\mathcal{U}^{r} \subset \mathcal{U}^{f}$. The vector of unknowns $\mathrm{u}^{f}$ can be then approximated

$$
\mathrm{u}^{f}=\mathrm{Q} \mathbf{u}^{r},
$$

where $\mathrm{Q}$ gathers discrete representations of $q_{i}(\boldsymbol{x})$ in $\mathcal{U}^{f}$ as its columns. Finally, we approximate the sought XFEM quantities with the fine-scale problem projected on a subspace as

$$
\underbrace{Q^{\top} K^{f} Q}_{\mathrm{K}^{r}} u^{r}=\underbrace{Q^{\top} \mathrm{f}^{f}}_{\mathrm{f}^{r}},
$$

which allows us to use standard routines for a stiffness matrix assembly and simplifies imposing Dirichlet boundary conditions by restricting the modes to unknown DOFs only. 


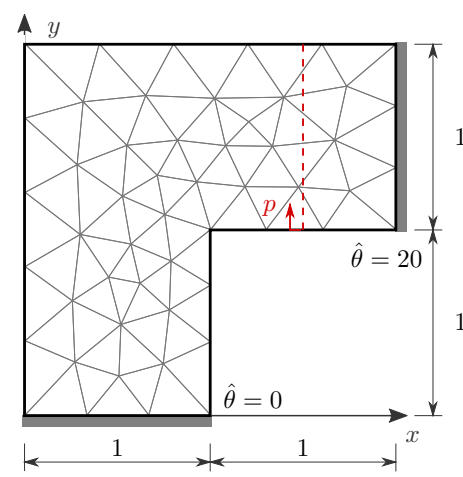

(a)

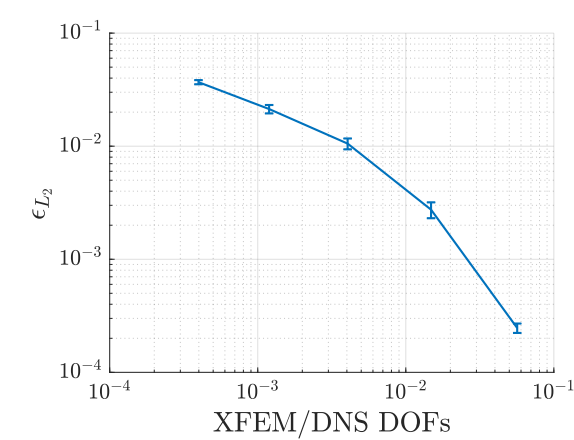

(b)

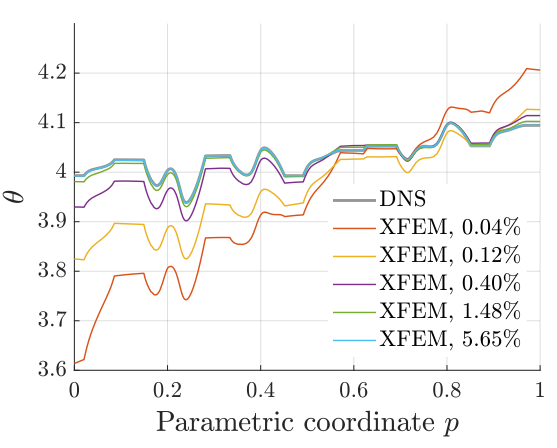

(c)

Fig. 2: Geometry and loading of the illustrative example (a), global errors (b), and local temperature profiles $(c)$.

\section{Results}

We illustrate the proposed method with an analysis of an L-shaped domain subjected to prescribed temperatures at the bottom and right-hand-side edges, see Fig. 2a. Microstructural geometry is assembled from the tiles depicted in Fig. 1b. Both material phases are isotropic with the conductivity of inclusions $\boldsymbol{K}=100 \boldsymbol{I}$ and the matrix $K=1 \boldsymbol{I}$. The initial macroscopic discretization is shown in Fig. 2a. The solution $\theta^{f}$ obtained for the high-dimensional $\mathcal{U}^{f}$ with quadratic Lagrangian elements is considered as the reference solution (DNS). Fig. 2b reports the overall relative error in $L_{2}$ norm for five uniformly refined macroscopic discretization. The error bars depict the standard deviation obtained for 50 different microstructure realizations. Fig. 2c shows the local temperature profile at the cross-section highlighted in Fig. 2a.

\section{Conclusions}

The methodology delivers acceptable error while significantly reducing the number of unknowns; Only $1.6 \%$ of DOFs were sufficient to obtain approximately $0.3 \%$ relative error compared to DNS. In addition, implementation of Eq. (7) holds promise for further acceleration by a low-rank approximation, such as the hyper-reduction method.

\section{Acknowledgments}

Martin Doškár and Jan Novák acknowledge the endowment of the Ministry of Industry and Trade of the Czech Republic under the project No. FV10202. Martin Doškář was partially supported by the Grant Agency of the Czech Technical University in Prague through project No. SGS18/036/OHK1/1T/11 "Modelling Heterogeneous Materials and Identification of Parameters of Macroscopic Material Models."

\section{References}

Doškář, M., Novák, J. and Zeman, J. (2014), Aperiodic compression and reconstruction of real-world material systems based on Wang tiles. Physical Review E, Vol. 90, No. 6, p 062118.

Novák, J., Kučerová, A. and Zeman, J.(2012) Compressing random microstructures via stochastic Wang tilings. Physical Review E, Vol. 86, No. 4, p 040104.

Novák, J., Kučerová, A. and Zeman, J.(2013) Microstructural enrichment functions based on stochastic Wang tilings. Modelling and Simulation in Materials Science and Engineering, Vol. 21, No. 2, p 025014.

Plews, J.A. and Duarte, C.A. (2015) Bridging multiple structural scales with a generalized finite element method. International Journal for Numerical Methods in Engineering, Vol. 102, No. 3-4, pp 180-201.

Strouboulis, T., Zhang, L. and Babuška, I. (2003) Generalized finite element method using mesh-based handbooks: application to problems in domains with many voids. Computer Methods in Applied Mechanics and Engineering Vol. 192, No. 28-30, pp 3109-3161. 